\title{
Serum Testosterone Level Can Be Predictive Factor for Upstaging in Clinically Localized Prostate Cancer
}

\author{
Soon Oh Kwon ${ }^{1}$, Kyeong-Hyeon Byeon ${ }^{1}$, Jae-Wook Chung ${ }^{1}$, Yun-Sok Ha ${ }^{1}$, \\ Seock Hwan Choi ${ }^{1}$, Bum Soo Kim ${ }^{1}$, Hyun Tae Kim${ }^{1}$, Tae-Hwan Kim, \\ Eun Sang Yoo', Ghil Suk Yoon ${ }^{2}$, Jun Nyung Lee ${ }^{1}$, Tae Gyun Kwon ${ }^{1,3}$

\footnotetext{
${ }^{1}$ Department of Urology, School of Medicine, Kyungpook National University, Daegu, Korea ${ }^{2}$ Department of Pathology, School of Medicine, Kyungpook National University, Daegu, Korea
} \\ ${ }^{3}$ Joint Institute for Regenerative Medicine, Kyungpook National University, Daegu, Korea
}

\begin{abstract}
Purpose: To determine an appropriate surgical technique, it is important to predict pathological results for patients with clinically localized prostate cancer ( $\mathrm{PCa}$ ) eligible for nerve-sparing radical prostatectomy (NSRP). Several studies have highlighted that serum testosterone level was associated with aggressive features of PCa. Therefore, we analyzed factors, including serum testosterone, to predict upstaging and upgrading after surgery for patients with clinically localized PCa eligible for NSRP.

Materials and Methods: We retrospectively evaluated patients who underwent radical prostatectomy (RP) between January 2015 and May 2018 at our institution. Patients with Gleason grade group 1 or 2 on biopsy, prostate-specific antigen $<10$, and $\leq$ clinical/radiologic stage T2 were included in this study. Upstaging and upgrading were defined as pathological stage $\geq T 3 a$ and Gleason grade group $\geq 3$, respectively. We evaluated the patients' demographics and outcomes according to upstaging and upgrading after surgery. Predictive factors for upstaging and upgrading were analyzed using a multivariate logistic regression model.

Results: Of 108 patients included in the study, upstaging and upgrading after surgery were observed in $24(22.2 \%)$ and $36(33.3 \%)$, respectively. Low serum testosterone level, small prostate size, and positive core number $\geq 3$ on biopsy were identified as predictive factors for upstaging in multivariate analysis. Although serum testosterone was associated with upgrading in univariate analysis, only clinical/radiologic stage and biopsy Gleason grade group were observed as predictive factors for upgrading in multivariate analysis.

Conclusions: Serum testosterone level was identified as a predictive factor for upstaging after RP for clinically localized PCa eligible for NSRP.

(Korean J Urol Oncol 2020;18:116-123)
\end{abstract}

Key Words: Pathology $\cdot$ Prostate cancer $\cdot$ Prostatectomy $\cdot$ Testosterone $\cdot$ Treatment outcome

Received March 17, 2020, Revised April 3, 2020, Accepted April 13, 2020

Comesponding Author: Jun Nyung Lee

Department of Urology, School of Medicine, Kyungpook National University, 807 Hoguk-ro, Buk-gu, Daegu 41404, Korea

E-mail: 1jnlover@gmail.com

Tel: +82-53-200-2675, Fax: +82-53-200-2027, ORCID: https://orcid.org/0000-0002-6342-9846

Co-comesponding Author: Tae Gyun Kwon

Department of Urology, School of Medicine, Kyungpook National University, 807 Hoguk-ro, Buk-gu, Daegu 41404, Korea

E-mail: tgkwon@knu.ac.kr

Tel: +82-53-420-5841, Fax: +82-53-200-2027, ORCID: https://orcid.org/0000-0002-4390-0952

- This research was supported by the Basic Science Research Program, through the National Research Foundation of Korea (NRF) and funded by the Korean government (MSIT), (2018R1C1B5040264) (2019R1A2C1004046) (2019R1F1A1044473) (2019R1H1A1079839).

(7) (1) This is an Open Access article distributed under the terms of the Creative Commons Attribution Non-Commercial License (http://creativecommons.org/licenses/by-nc/4.0/) which permits unrestricted non-commercial use, distribution, and reproduction in any medium, provided the original work is properly cited.

2020 (C) Copyright The Korean Urological Oncology Society and The Korean Prostate Society. All Rights Reserved. 


\section{INTRODUCTION}

Prostate cancer $(\mathrm{PCa})$ is among the most common male cancers worldwide, and its incidence is gradually increasing. ${ }^{1}$ Several treatment modalities, including active surveillance (AS), radical prostatectomy (RP), radiation therapy, and androgen deprivation therapy, have been established for patients with clinically localized PCa. Generally, RP has been considered as the gold standard definitive treatment for clinically localized PCa. However, RP can cause surgical complications, including urinary incontinence and erectile dysfunction, which can adversely influence quality of life. ${ }^{2,3}$ To mitigate such complications, nerve-sparing radical prostatectomy (NSRP), which preserves the neurovascular bundles, can apply to patients with selected criteria based on preoperative evaluations, such as prostate-specific antigen (PSA), clinical stage, and biopsy grade.,

Although NSRP is currently acceptable for selected patients with $\mathrm{PCa}$, it can sometimes cause incomplete tumor excision, depending on the tumor stage. ${ }^{5}$ Additionally, recent studies have shown that pathological stage and Gleason grade after RP are often inconsistent with clinical stage and biopsy Gleason grade. ${ }^{6}$ The discrepancy between preoperative parameters and pathological results is usually observed as upstaging or upgrading. ${ }^{6-11}$ Therefore, it is necessary to determine predictive factors for worse pathological outcomes, such as upgrading and upstaging, relative to preoperative findings among patients with $\mathrm{PCa}$ amenable to NSRP.

Several studies have revealed that preoperative serum testosterone level is associated with aggressive features of $\mathrm{PCa}$, such as high stage and high grade. ${ }^{12-16}$ Additionally, a recent study showed that low serum testosterone $(<300$ $\mathrm{ng} / \mathrm{dL}$ ) was associated with a high rate of upgrading and upstaging after RP. ${ }^{8}$ Another study also reported that low serum testosterone was associated with a positive surgical margin in RP specimens. ${ }^{17}$

In this study, we evaluated factors, including preoperative serum testosterone, to predict upstaging and upgrading after NSRP for PCa.

\section{MATERIALS AND METHODS}

We retrospectively reviewed $\mathrm{PCa}$ patients who underwent NSRP, performed by a single experienced surgeon between January 2015 and May 2018 at Kyungpook National University Chilgok Hospital. Patients with biopsy Gleason grade group 1 or $2, \mathrm{PSA}<10 \mathrm{ng} / \mathrm{mL}$, and clinical/radiologic stage $\leq$ T2 (using the 2009 TNM staging system) on preoperative evaluations were included in this study. We excluded patients who underwent neoadjuvant therapy, such as radiotherapy or hormonal therapy, and those with known medical problems that might affect testosterone status, such as thyroid disease, liver disease, and hypoalbuminemia. Patients with a history of $5 \alpha$ RI medication administration or testosterone replacement therapy were also excluded.

All patients were diagnosed with PCa by 12 -core transrectal needle prostatic biopsies at our institution and underwent multiparametric magnetic resonance imaging for preoperative radiologic staging.

All patients underwent open RP or robot-assisted RP according to preoperative counseling. Open RP or robot-assisted RP were conducted in an ante-grade fashion using a nerve-sparing technique and a continuous urethrovesical anastomosis suture in the manner previously reported. ${ }^{18}$ Gleason grade was assessed according to the 2014 International Society of Urological Pathology Modified Gleason System. ${ }^{19}$ Prostate volume was measured by standard methods using transrectal ultrasonography, and PSA density (PSAD) was calculated by dividing PSA with prostate volume. Using an immunoassay at our institution's laboratory, the preoperative serum testosterone level (ng/dL) was measured. Considering diurnal fluctuations of the testosterone level, serum samples were collected in the morning between $8 \mathrm{AM}$ and $10 \mathrm{AM}$ when testosterone levels are high and stable.

The upstaging and upgrading were defined as nonorgan confined disease (pathological stage $\geq \mathrm{T} 3 \mathrm{a}$ ) and pathological Gleason grade group $\geq 3$ after RP, respectively. We evaluated the incidence of upstaging and upgrading after RP in patients with biopsy Gleason grade group 1 or $2, \operatorname{PSA}<10$ $\mathrm{ng} / \mathrm{mL}$, and clinical/radiologic stage $\leq \mathrm{T} 2$ on preoperative evaluation.

We also compared the patients' characteristics according 
to upstaging and upgrading using Student t-test and the chi-square test. Predictive factors for upstaging and upgrading after RP were analyzed using a multivariate logistic regression model. Statistical analyses were performed using IBM SPSS Statistics ver. 23.0 (IBM Co., Armonk, NY, USA), and statistical significance was established with p-value of $<0.05$.

The present study protocol was reviewed and approved by the Institutional Review Board of Kyungpook National University Chilgok Hospital (Reg. No. KNUMC 2019-12-0 21). Informed consent was submitted by all subjects when they were enrolled.

\section{RESULTS}

A total of 108 patients were included in the study. The mean age was $65.5 \pm 6.2$ years, mean preoperative PSA was $6.3 \pm 1.8 \mathrm{ng} / \mathrm{mL}$, and mean preoperative serum testosterone was $347.2 \pm 154.1 \mathrm{ng} / \mathrm{dL}$. Clinical/radiologic $\mathrm{T}$ stage was cT1c for 18 patients (16.7\%) and cT2 for 90 patients $(83.3 \%)$. Sixty-nine patients $(63.9 \%)$ were classified as Gleason grade group 1, and 39 patients $(36.1 \%)$ were classified as grade group 2. Table 1 shows the clinical and pathological characteristics of patients included in this study. Upstaging and upgrading after surgery were observed in $22.2 \%$ (24 of 108) and 33.3\% (36 of 108) patients, respectively. Among the 24 patients in the upstaging group, 20 $(83.3 \%)$ were upstaged to pT3a, and $4(16.7 \%)$ were upstaged to pT3b. Among the 36 patients who were upgraded, $30(83.3 \%)$ were upgraded to Gleason grade group 3, and $6(16.7 \%)$ were upgraded to Gleason grade group 4 (Fig. 1).

Table 2 shows patients' characteristics according to upstaging and upgrading status. Mean prostate size was smaller and PSA density was higher among patients who were upstaged compared with those who were not $(25.5 \pm 5.5$ vs. $34.5 \pm 10.8, \mathrm{p}<0.001$ and $0.28 \pm 0.13$ vs. $0.20 \pm 0.09, \mathrm{p}=0.012$, respectively). Mean preoperative serum testosterone was significantly lower in the upstaged patients compared with those who were not upstaged $(284.6 \pm 108.8$ vs. $367.6 \pm 156.8$, $\mathrm{p}=0.017$ ). The proportion of patients with a positive core number $\geq 3$ at biopsy was higher in the upstaged group compared with the nonupstaged group (75.0\% vs. $35.7 \%$, $\mathrm{p}=0.001$ ). There were no significant differences in age, body mass index, PSA, biopsy Gleason grade group, or operative method (robot-assisted RP vs. open RP) according upstaging status after RP.

In terms of upgrading status, mean preoperative serum testosterone level was significantly lower among upgraded patients compared with those who were not upgraded (308.4 \pm 106.9 vs. $369.5 \pm 165.7, p=0.047)$. In addition, biopsy Gleason grade group and clinical/radiologic stage were significantly different between patients who were upgraded and those who were not $(\mathrm{p}=0.034$ and $\mathrm{p}=0.006$, respectively). Other variables did not show any significance.

The multivariate logistic regression model revealed that preoperative serum testosterone level (odds ratio [OR],

Table 1. Clinical and pathological characteristics of patients included in the study $(\mathrm{n}=108)$

\begin{tabular}{lc}
\hline \multicolumn{1}{c}{ Characteristic } & Value \\
\hline Age (yr) & $65.5 \pm 6.2$ \\
Body mass index $\left(\mathrm{kg} / \mathrm{m}^{2}\right)$ & $24.3 \pm 2.9$ \\
PSA $(\mathrm{ng} / \mathrm{mL})$ & $6.3 \pm 1.8$ \\
Size $(\mathrm{mL})$ & $32.2 \pm 10.9$ \\
PSAD & $0.5 \pm 3.1$ \\
Testosterone level (ng/dL) & $347.2 \pm 154.1$ \\
Total positive core number & $3.1 \pm 2.3$ \\
Positive core number & \\
$\geq 3$ & $48(44.4)$ \\
$<3$ & $60(55.6)$ \\
Biopsy Gleason grade & $69(63.9)$ \\
Group 1 & $39(36.1)$ \\
Group 2 & \\
Pathological Gleason grade & $13(12.0)$ \\
Group 1 & $59(54.6)$ \\
Group 2 & $30(27.8)$ \\
Group 3 & $6(5.6)$ \\
Group 4 & \\
Clinical/radiologic stage & $18(16.7)$ \\
T1c & $90(83.3)$ \\
T2 & $84(77.8)$ \\
Pathological stage & $20(18.5)$ \\
pT2 & $4(3.7)$ \\
pT3a & \\
pT3b & \\
Operative method & $(21.3)$ \\
RARP & \\
Open RP & \\
\hline
\end{tabular}

Values are presented as mean \pm standard deviation or number (\%). PSA: prostate-specific antigen, PSAD: prostate-specific antigen density, RARP: robot-assisted radical prostatectomy, RP: radical prostatectomy. 
A

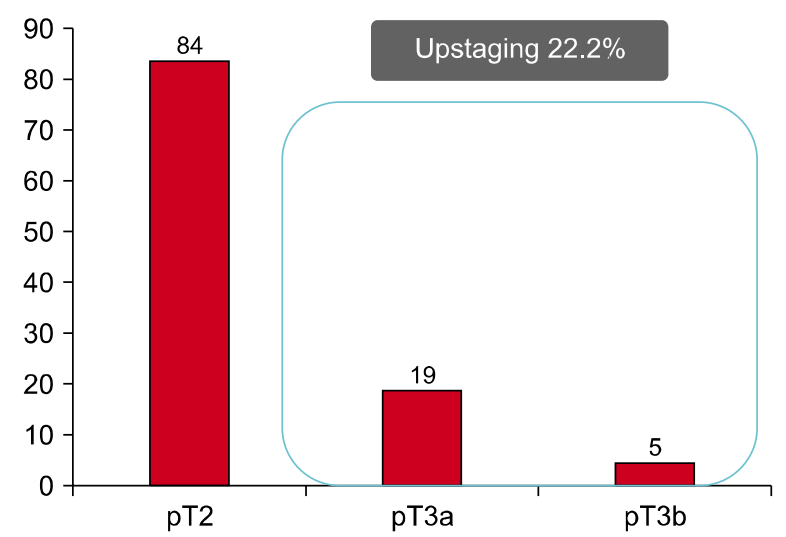

B

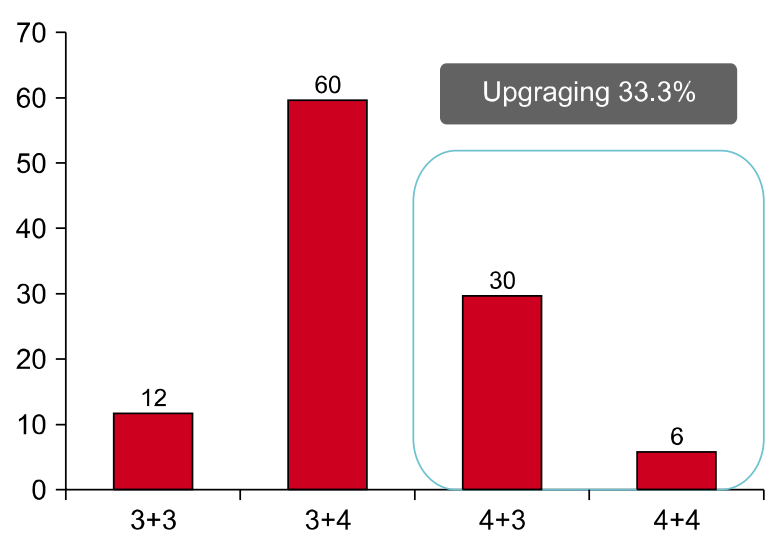

Fig. 1. The rates of upstaging (A) and upgrading (B) after surgery.

Table 2. Comparison of variables according to upstaging and upgrading after surgery

\begin{tabular}{|c|c|c|c|c|c|c|}
\hline \multirow{2}{*}{ Variable } & \multicolumn{3}{|c|}{ Upstaging } & \multicolumn{3}{|c|}{ Upgrading } \\
\hline & Yes $(n=24)$ & No $(n=84)$ & p-value & Yes $(n=36)$ & No $(n=72)$ & p-value \\
\hline Age (yr) & $66.7 \pm 4.4$ & $65.2 \pm 6.7$ & 0.216 & $65.7 \pm 4.8$ & $65.4 \pm 6.9$ & 0.828 \\
\hline BMI $\left(\mathrm{kg} / \mathrm{m}^{2}\right)$ & $24.4 \pm 3.3$ & $24.3 \pm 2.7$ & 0.926 & $24.5 \pm 2.6$ & $24.2 \pm 3.0$ & 0.628 \\
\hline PSA $(\mathrm{ng} / \mathrm{mL})$ & $6.49 \pm 2.0$ & $6.26 \pm 1.7$ & 0.616 & $6.15 \pm 1.6$ & $6.42 \pm 1.8$ & 0.432 \\
\hline Size $(\mathrm{mL})$ & $25.5 \pm 5.5$ & $34.5 \pm 10.8$ & $<0.001$ & $33.4 \pm 11.7$ & $32.0 \pm 10.0$ & 0.529 \\
\hline PSAD & $0.28 \pm 0.13$ & $0.20 \pm 0.09$ & 0.012 & $0.21 \pm 0.10$ & $0.22 \pm 0.11$ & 0.378 \\
\hline Testosterone level (ng/dL) & $284.6 \pm 108.8$ & $367.6 \pm 156.8$ & 0.017 & $308.4 \pm 106.9$ & $369.5 \pm 165.7$ & 0.047 \\
\hline Total positive core number & $3.8 \pm 2.1$ & $2.9 \pm 2.3$ & 0.733 & $2.9 \pm 2.0$ & $3.2 \pm 2.4$ & 0.564 \\
\hline Positive core number & & & 0.001 & & & 1.000 \\
\hline$\geq 3$ & 18 & 30 & & 16 & 32 & \\
\hline$<3$ & 6 & 54 & & 20 & 40 & \\
\hline Biopsy Gleason grade & & & 0.108 & & & 0.034 \\
\hline Group 1 & 12 & 57 & & 18 & 51 & \\
\hline Group 2 & 12 & 27 & & 18 & 21 & \\
\hline Clinical/radiologic stage & & & 0.758 & & & 0.006 \\
\hline $\mathrm{T} 1 \mathrm{c}$ & 3 & 15 & & 1 & 17 & \\
\hline $\mathrm{T} 2$ & 21 & 69 & & 35 & 55 & \\
\hline Operative method & & & 0.286 & & & 0.406 \\
\hline RARP & 17 & 68 & & 30 & 55 & \\
\hline Open RP & 7 & 16 & & 6 & 17 & \\
\hline
\end{tabular}

Values are presented as mean \pm standard deviation or number.

BMI: body mass index, PSA: prostate-specific antigen, PSAD: prostate-specific antigen density, RARP: robot-assisted radical prostatectomy, RP: radical prostatectomy.

0.993; 95\% confidence interval $[\mathrm{CI}], 0.987-0.999 ; \mathrm{p}=$ 0.034), prostate size (OR, 0.765; 95\% CI, 0.669-0.875; $\mathrm{p}<0.001$ ), and a positive core number $\geq 3$ at biopsy (OR, 9.856; 95\% CI, 2.230-43.557; $\mathrm{p}=0.003$ ) were associated with upstaging after RP (Table 3). The cutoff value for preoperative serum testosterone level for upstaging was 273.7 ng/dL (sensitivity, 71.3\%; specificity, 66.7\%, as determined by receiver operating curve analysis) (Fig. 2). In addition, clinical/radiologic stage (T2 vs. T1c) and Gleason grade group at biopsy (2 vs. 1) were significantly associated with upgrading in the multivariate analysis (OR, 11.260; 95\% CI, 1.410-89.914; $\mathrm{p}=0.022$ and OR, 2.526; 95\% CI, $1.063^{-}$ 5.998; $\mathrm{p}=0.036$, respectively). 
Table 3. Factors associated with upstaging and upgrading by multivariable logistic regression

\begin{tabular}{lcccccc}
\hline \multirow{2}{*}{ Variable } & \multicolumn{2}{c}{ Upstaging } & & & \multicolumn{2}{c}{ Upgrading } \\
\cline { 2 - 3 } & OR $(95 \%$ CI $)$ & p-value & & OR (95\% CI) & p-value \\
\hline Testosterone level (continuous) & $0.993(0.987-0.999)$ & 0.034 & & $0.997(0.994-1.000)$ & 0.072 \\
Prostate size (continuous) & $0.765(0.669-0.875)$ & $<0.001$ & & - & - \\
Positive core number $\geq 3$ vs $<3$ & $9.856(2.230-43.557)$ & 0.003 & & - & - \\
Clinical/radiologic stage T2 vs. T1c & - & - & & $11.260(1.410-89.914)$ & 0.022 \\
Biopsy Gleason grade group 2 vs. 1 & - & & $2.526(1.063-5.998)$ & 0.036 \\
\hline
\end{tabular}

OR: odds ratio, $\mathrm{CI}$ : confidence interval.

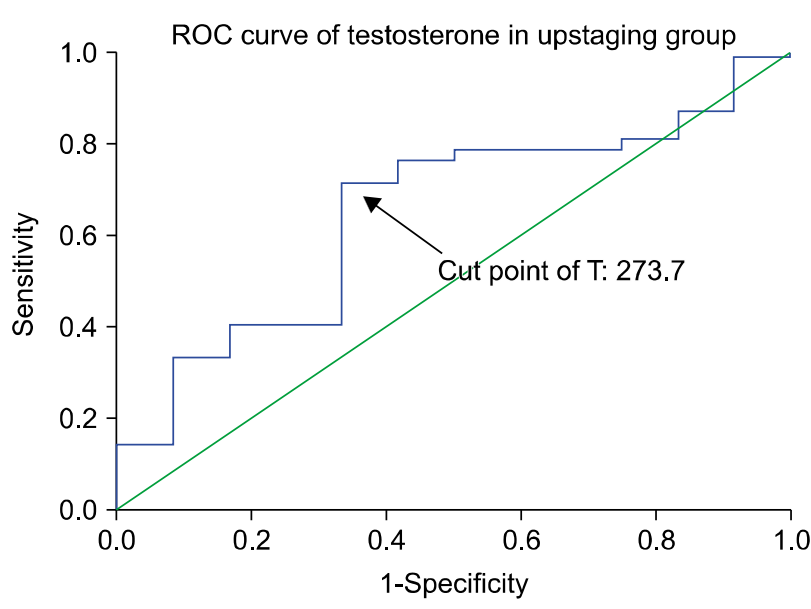

Fig. 2. Receiver-operating characteristic (ROC) curve of preoperative serum testosterone level for upstaging after surgery. Cutoff point for testosterone level was $273.7 \mathrm{ng} / \mathrm{dL}$ (sensitivity, $71.3 \%$; specificity, $66.7 \%$; area under the curve, 0.649 ) on the ROC curve analysis.

\section{DISCUSSION}

Currently, NSRP is a widely accepted surgical method for select patients with clinically localized $\mathrm{PCa}$, to minimize postoperative complications, including erectile dysfunction and postprostatectomy incontinence. However, postoperative upstaging and upgrading-which might have altered the operative technique selection if they had been predicted-are not uncommon after NSRP. Recently, several studies revealed that preoperative serum testosterone reflects tumor aggressiveness among PCa patients. ${ }^{12-16}$ Therefore, we assessed the incidence and predictive factors for upstaging and upgrading among PCa patients after NSRP at our institution. We observed significant incidences of upstaging $(22.2 \%)$ and upgrading (33.3\%). Furthermore, preoperative serum testosterone was negatively associated with upstaging and upgrading after RP. Multivariate analysis revealed preoperative serum testosterone as a significant predictor upstaging, despite its lack of a significant association with postoperative upgrading.

The key objectives of RP for PCa include ensuring good postoperative erectile function and urinary continence without compromising oncological outcomes. Techniques that emphasize the preservation of neurovascular bundles during RP have been developed to minimize postoperative complications for appropriately selected patients. ${ }^{3}$ Several studies have confirmed the benefits of NSRP for the recovery of erectile function and urinary continence. ${ }^{20-23}$ While nervesparing techniques have several advantages in terms of quality of life, dissection closer to the prostate capsule during NSRP may increase the risk of incising the tumor or incomplete tumor excision, resulting in a positive surgical margin, and may increase risk of biochemical progression and cancer recurrence. ${ }^{5}$

When choosing the treatment method, particularly when considering NSRP, the surgeon most strongly considered the index PSA, clinical stage, and the biopsy Gleason grade group. However, recent research has shown that pathological stage and grade after surgery are often inconsistent with clinical stage and biopsy grade, and this is usually expressed as upgrading or upstaging. ${ }^{6-11}$ For patients whose real pathological stage and grade exceed the clinical stage and biopsy grade, the selected surgical method-nerve-sparing surgery, for example-could underestimate the aggressiveness of the $\mathrm{PCa}$ and compromise cancer control. Therefore, it is necessary to identify predictive factors for upstaging and upgrading for selecting a proper treatment strategy or surgical method for PCa patients for whom there are several options.

Several recent studies have focused on determining pre- 
dictors of upstaging and upgrading among PCa patients. ${ }^{6-11}$ In a large cohort study including 7,643 patients who underwent RP, $36.3 \%$ of patients had their needle biopsy Gleason score upgraded from 5 or 6 to a higher grade after RP. ${ }^{6}$ Sooriakumaran et al. ${ }^{10}$ reported that $40.4 \%$ patients were either upstaged $(3.9 \%)$ or upgraded $(39.6 \%)$ after RP among 750 patients with low-risk PCa, clinically eligible for AS. In a Korean multicenter study with 324 RP specimens from low-risk PCa patients, upstaging and upgrading were observed in $9.6 \%$ and $43.8 \%$ samples, respectively. ${ }^{9}$ In another study of Korean PCa patients who were eligible for AS (clinical stage T1c/T2a, PSA level $10 \mathrm{ng} / \mathrm{mL}$ or less, and Gleason score 6 or less), upstaging and upgrading were observed in $11.0 \%$ and $57.8 \%$ of 593 patients, respectively. ${ }^{11}$ A recent study with similar inclusion criteria (biopsy Gleason score $\leq 6$, clinical stage $\leq \mathrm{T} 2 \mathrm{c}$, and PSA $<10 \mathrm{ng} / \mathrm{mL}$ ) to our study revealed that upstaging occurred in $43.7 \%$, and upgrading occurred in $37.1 \%$ of 167 patients. $^{8}$ Unlike previous studies, however, our study showed relatively high upstaging (33.3\%) and low upgrading (22.2\%). We assumed that this discrepancy from previous research was caused by differences in the participant characteristics and radiologic staging using multiparametric magnetic resonance imaging in the present study. This study was conducted on patients eligible for NSRP (biopsy Gleason grade group 1 or 2, $\mathrm{PSA}<10$, and clinical/radiologic stage $\leq \mathrm{T} 2$ ) at our institution, while previous studies investigated patients who were eligible for AS.

In the present study, preoperative serum testosterone was negatively correlated with upstaging and upgrading after $\mathrm{RP}$, and it was identified as a predictive factor for upstaging in multivariate analysis. Although it is still controversial, testosterone has been widely evaluated as a predictive factor for worse pathological outcomes, such as upstaging and upgrading, and oncologic outcomes. ${ }^{13-17,24,25}$ Previous studies have suggested that serum testosterone level is inversely correlated with tumor aggressiveness, which is a descriptor that encompasses both high stage ${ }^{13,14}$ and high grade. ${ }^{15,16}$ Teloken et al. ${ }^{17}$ reported that low serum testosterone levels were associated with positive surgical margins after RP, while not with PSA levels, Gleason score (biopsy or specimen), pathological stage, or capsular perforation. Moreover, Asian studies have identified preoperative serum testosterone level as an independent predictor of biochemical re- currence after RP among patients with clinically localized $\mathrm{PCa}^{24,25}$

Recently, several reports have highlighted the association of low serum testosterone levels with upstaging and upgrading after RP among patients with localized PCa. ${ }^{8,12}$ Gao et al. ${ }^{8}$ investigated the association between serum testosterone and upgrading or upstaging among PCa patients with biopsy Gleason scores $\leq 6$, clinical stage $\leq \mathrm{T} 2 \mathrm{c}$, and PSA $<10 \mathrm{ng} / \mathrm{mL}$. They confirmed preoperative serum testosterone level as a unique independent predictor of pathological upstaging and upgrading after RP. Moreover, Ferro et al. ${ }^{12}$ assessed the impact of serum testosterone for predicting unfavorable outcomes among $\mathrm{PCa}$ patients eligible for AS, which is currently considered acceptable for clinically localized PCa. They suggested that patients with hypogonadism were not eligible for AS because low serum testosterone is associated with unfavorable pathological outcomes, including upstaging, upgrading, and positive surgical margins. Our findings also supported the clinical impact of low serum testosterone as a predictive factor of upstaging and upgrading among patients with localized PCa eligible for NSRP on preoperative evaluation. Therefore, surgeons should be cautious when selection of a treatment course and proceeding with NSRP for eligible patients with low serum testosterone.

Recent studies, including the present study, have demonstrated that serum testosterone level is predictive of tumor aggressiveness, including the potential for upstaging and upgrading. Although the exact mechanism is not yet fully understood, it may be assumed that involves the inhibition of testosterone by highly aggressive prostate tumors and negative feedback control of pituitary gonadotropin secretion. ${ }^{8}$ Moreover, metabolic disorders that are associated with hypogonadism might contribute to unfavorable $\mathrm{PCa}$ outcomes. $^{12,26,27}$

In this study, small prostate size and positive core number $\geq 3$ on biopsy were also identified as predictive factors for upstaging in multivariate analysis. Previous studies have shown small prostate size and high positive core number on prostate biopsy to be associated with aggressive features of PCa. ${ }^{9,11,28-30}$. Similar with our results, 2 Korean studies found that prostate volume and positive core number were significantly associated with upstaging ${ }^{11}$ or worsening prognosis $^{9}$ in multivariate analyses. Additionally, a Japanese study $^{29}$ and a Swedish study ${ }^{30}$ also reported that smaller 
prostate volume was associated with adverse pathology. Regarding the mechanism by which prostate volume and positive core number are predictors of $\mathrm{PCa}$ aggressiveness, they might reflect a relatively larger tumor burden with a high probability of disease that has progressed beyond the prostate capsule.

Several limitations of our study should be considered. First, this was a retrospective analysis of the records of a relatively small sample of patients treated at a single institution. Although the criteria for NSRP eligibility were followed in this study, selection bias regarding the surgical indication was unavoidable. Additionally, we did not access to data reflecting long-term oncologic outcomes, such as biochemical recurrence and metastasis-free survival. These limitations highlight the need for more standardized study designs and outcome reporting methods in the future. Furthermore, additional studies are necessary to elucidate the underlying mechanisms involving serum testosterone in relation to $\mathrm{PCa}$. Although this retrospective study had several limitations, it demonstrated that low preoperative serum testosterone level, small prostate size, and positive core number $\geq 3$ on biopsy should be considered valuable predictors of upstaging after RP in clinically localized PCa amenable to NSRP. We hope that the results of this study can help clinicians develop appropriate management strategies and choose appropriate surgical methods to treat clinically localized PCa.

\section{CONCLUSIONS}

Low preoperative serum testosterone level, small prostate size, and positive biopsy core number $\geq 3$ were identified as predictive factors for upstaging after RP in clinically localized PCa eligible for nerve-sparing surgery. Therefore, preoperative serum testosterone should be measured and considered as a predictor of non-organ confined disease in patients with clinically localized PCa eligible for nerve-sparing surgery.

\section{CONFLICT OF INTEREST}

The authors claim no conflicts of interest.

\section{REFERENCES}

1. Jung KW, Won YJ, Kong HJ, Oh CM, Cho H, Lee DH, et al. Cancer statistics in Korea: incidence, mortality, survival, and prevalence in 2012. Cancer Res Treat 2015;47: 127-41.

2. Mandel P, Graefen M, Michl U, Huland H, Tilki D. The effect of age on functional outcomes after radical prostatectomy. Urol Oncol 2015;33:203.e11-8.

3. Mottet N, Bellmunt J, Bolla M, Briers E, Cumberbatch MG, De Santis M, et al. EAU-ESTRO-SIOG guidelines on prostate cancer. Part 1: screening, diagnosis, and local treatment with curative intent. Eur Urol 2017;71:618-29.

4. Michl U, Tennstedt P, Feldmeier L, Mandel P, Oh SJ, Ahyai $S$, et al. Nerve-sparing surgery technique, not the preservation of the neurovascular bundles, leads to improved long-term continence rates after radical prostatectomy. Eur Urol 2016;69:584-9.

5. Catalona WJ, Bigg SW. Nerve-sparing radical prostatectomy: evaluation of results after 250 patients. J Urol 1990; 143:538-43.

6. Epstein JI, Feng Z, Trock BJ, Pierorazio PM. Upgrading and downgrading of prostate cancer from biopsy to radical prostatectomy: incidence and predictive factors using the modified Gleason grading system and factoring in tertiary grades. Eur Urol 2012;61:1019-24.

7. Fine SW, Epstein JI. A contemporary study correlating prostate needle biopsy and radical prostatectomy Gleason score. J Urol 2008;179:1335-8.

8. Gao Y, Jiang CY, Mao SK, Cui D, Hao KY, Zhao W, et al. Low serum testosterone predicts upgrading and upstaging of prostate cancer after radical prostatectomy. Asian J Androl 2016;18:639-43.

9. Hwang I, Lim D, Jeong YB, Park SC, Noh JH, Kwon DD, et al. Upgrading and upstaging of low-risk prostate cancer among Korean patients: a multicenter study. Asian J Androl 2015;17:811-4.

10. Sooriakumaran P, Srivastava A, Christos P, Grover S, Shevchuk M, Tewari A. Predictive models for worsening prognosis in potential candidates for active surveillance of presumed low-risk prostate cancer. Int Urol Nephrol 2012; 44:459-70.

11. Jeon HG, Yoo JH, Jeong BC, Seo SI, Jeon SS, Choi HY, et al. Comparative rates of upstaging and upgrading in Caucasian and Korean prostate cancer patients eligible for active surveillance. PLoS One 2017;12:e186026.

12. Ferro $M$, Lucarelli $G$, Bruzzese $D$, Di Lorenzo $G$, Perdonà $\mathrm{S}$, Autorino R, et al. Low serum total testosterone level as a predictor of upstaging and upgrading in low-risk prostate cancer patients meeting the inclusion criteria for active 
surveillance. Oncotarget 2017;8:18424-34.

13. Imamoto $\mathrm{T}$, Suzuki H, Fukasawa $\mathrm{S}$, Shimbo M, Inahara M, Komiya A, et al. Pretreatment serum testosterone level as a predictive factor of pathological stage in localized prostate cancer patients treated with radical prostatectomy. Eur Urol 2005;47:308-12.

14. Isom-Batz G, Bianco FJ Jr, Kattan MW, Mulhall JP, Lilja $\mathrm{H}$, Eastham JA. Testosterone as a predictor of pathological stage in clinically localized prostate cancer. J Urol 2005; 173:1935-7.

15. Schatzl G, Madersbacher S, Thurridl T, Waldmüller J, Kramer G, Haitel A, et al. High-grade prostate cancer is associated with low serum testosterone levels. Prostate 2001;47:52-8.

16. Pichon A, Neuzillet Y, Botto H, Raynaud JP, Radulescu C, Molinié V, et al. Preoperative low serum testosterone is associated with high-grade prostate cancer and an increased Gleason score upgrading. Prostate Cancer Prostatic Dis 2015;18:382-7.

17. Teloken C, Da Ros CT, Caraver F, Weber FA, Cavalheiro AP, Graziottin TM. Low serum testosterone levels are associated with positive surgical margins in radical retropubic prostatectomy: hypogonadism represents bad prognosis in prostate cancer. J Urol 2005;174:2178-80.

18. Kwon SY, Lee JN, Ha YS, Choi SH, Kim TH, Kwon TG. Open radical prostatectomy reproducing robot-assisted radical prostatectomy: involving antegrade nerve sparing and continuous anastomosis. Int Braz J Urol 2017;43:1043-51.

19. Epstein JI, Egevad L, Amin MB, Delahunt B, Srigley JR, Humphrey PA, et al. The 2014 International Society of Urological Pathology (ISUP) Consensus Conference on Gleason Grading of Prostatic Carcinoma: definition of grading patterns and proposal for a new grading system. Am J Surg Pathol 2016;40:244-52.

20. Murphy DG, Costello AJ. How can the autonomic nervous system contribute to urinary continence following radical prostatectomy? A "boson-like" conundrum. Eur Urol 2013; 63:445-7.

21. Reeves F, Preece P, Kapoor J, Everaerts W, Murphy DG, Corcoran NM, et al. Preservation of the neurovascular bundles is associated with improved time to continence af- ter radical prostatectomy but not long-term continence rates: results of a systematic review and meta-analysis. Eur Urol 2015;68:692-704.

22. Steineck G, Bjartell A, Hugosson J, Axén E, Carlsson S, Stranne J, et al. Degree of preservation of the neurovascular bundles during radical prostatectomy and urinary continence 1 year after surgery. Eur Urol 2015;67:559-68.

23. Narayan P, Konety B, Aslam K, Aboseif S, Blumenfeld W, Tanagho E. Neuroanatomy of the external urethral sphincter: implications for urinary continence preservation during radical prostate surgery. J Urol 1995;153:337-41.

24. Yamamoto S, Yonese J, Kawakami S, Ohkubo Y, Tatokoro M, Komai Y, et al. Preoperative serum testosterone level as an independent predictor of treatment failure following radical prostatectomy. Eur Urol 2007;52:696-701.

25. Kim HJ, Kim BH, Park CH, Kim CI. Usefulness of preoperative serum testosterone as a predictor of extraprostatic extension and biochemical recurrence. Korean J Urol 2012;53:9-13.

26. Freedland SJ, Isaacs WB, Mangold LA, Yiu SK, Grubb KA, Partin AW, et al. Stronger association between obesity and biochemical progression after radical prostatectomy among men treated in the last 10 years. Clin Cancer Res 2005;11:2883-8.

27. Iremashvili V, Soloway MS, Rosenberg DL, Manoharan M. Clinical and demographic characteristics associated with prostate cancer progression in patients on active surveillance. J Urol 2012;187:1594-9.

28. Liu JJ, Brooks JD, Ferrari M, Nolley R, Presti JC Jr. Small prostate size and high grade disease--biology or artifact? J Urol 2011;185:2108-11.

29. Yashi M, Mizuno T, Yuki H, Masuda A, Kambara T, Betsunoh $\mathrm{H}$, et al. Prostate volume and biopsy tumor length are significant predictors for classical and redefined insignificant cancer on prostatectomy specimens in Japanese men with favorable pathologic features on biopsy. BMC Urol 2014; $14: 43$.

30. Vellekoop A, Loeb S, Folkvaljon Y, Stattin P. Population based study of predictors of adverse pathology among candidates for active surveillance with Gleason 6 prostate cancer. J Urol 2014;191:350-7. 